\title{
Variables macroeconómicas e ingresos operacionales de las empresas colombianas: sectores representativos
}

\author{
Yánez Contreras, Martha Alicia* \\ Angulo Pico, Grace Margarita**
}

\begin{abstract}
Resumen
El presente artículo tiene como objetivo explicar cómo el entorno macroeconómico, puede ser un factor determinante del comportamiento de los ingresos operacionales de las empresas de los sectores más representativos de Colombia. La metodología empleada fue panel de datos con efectos fijos o aleatorios, según el resultado de la prueba correspondiente. Se analizaron los sectores energía, químico; construcción; comercio y otros servicios, entre 1998-2009. Los resultados mostraron que las variables macroeconómicas más influyentes en el desempeño de los ingresos corporativos son la tasa de interés y variación del índice de tasa de cambio real en los sectores químico y otros servicios; la tasa de desempleo en los sectores energía y construcción; y la tasa de inflación en el sector agropecuario. Pese a que las empresas del sector comercio no mostraron significancia estadística ante las variables macroeconómicas; el desempeño del PIB y la variación de la tasa de cambio real pueden determinar sus ingresos. En términos generales se verificó la respuesta diferencial de las empresas colombianas al contexto económico predominante a nivel departamental y nacional.
\end{abstract}

Palabras clave: Entorno macroeconómico, ingresos empresariales, sectores representativos, Colombia.

Recibido: 20-01-12. Aceptado: 18-05-13

* Magíster en Economía y en Economía del Medio Ambiente y los Recursos Naturales de la Universidad de los Andes, Colombia. Docente de tiempo completo y miembro del Grupo Mercado Laboral de la Universidad de Cartagena, Colombia, e-mail: myanezc@unicartagena.edu.co. yanezmartha@hotmail.com

** Economista de la Universidad de Cartagena. Joven Investigador del Grupo Mercado Laboral de la misma institución, e-mail: gangulop@unicartagena.edu.co. 


\title{
Macroeconomic Variables and Operations Income in Colombian Companies: Representative Sectors
}

\begin{abstract}
The objective of this article is to explain how the macroeconomic environment can be a determining factor in operations income behavior for companies in the most representative sectors of Colombia. The methodology used was the data panel with fixed or random effects, according to the result of the corresponding test. Energy, chemical, construction, commercial and other sectors were analyzed between 1998 and 2009. Results show that the macroeconomic variables most influential in the performance of corporate income are: the interest rate and variation of the real exchange rate in the chemical and other sectors; the unemployment rate in energy and construction sectors; and the inflation rate in the agricultural sector. Although enterprises in the commercial sector did not show statistical significance in terms of macroeconomic variables, GNP performance and variation in the real exchange rate can determine their income. In general terms, the differential response of Colombian companies was verified in the economic context, predominantly on departmental and national levels.
\end{abstract}

Keywords: Macroeconomic environment, entrepreneurial income, representative sectors, Colombia.

\section{Introducción}

Siempre ha existido la necesidad de identificar los factores que inciden en el desempeño de los ingresos operacionales de las empresas. La literatura económica ha centrado sus esfuerzos en factores micro y mesoeconómicos, olvidando que muchas veces los resultados logrados por las empresas son más la respuesta a variables de política macroeconómica, que la consecuencia de esfuerzos optimizadores internos. Por tal motivo, en el presente trabajo se plantearán algunas pautas para determinar el impacto del entorno macroeconómico sobre el desempeño de los ingresos operacionales de las empresas colombianas entre 1998 y 2009.

Esta necesidad de endogenizar el comportamiento de las variables macroe- conómicas en el desempeño de los ingresos operativos de las firmas, se debe en gran parte a la escasez de antecedentes investigativos nacionales e internacionales, referente a la temática. En Colombia, particularmente, son pocos los estudios que se tienen respecto a la materia. Por tanto, esta investigación es importante porque utiliza variables macroeconómicas para explicar el desempeño operacional de las empresas de los sectores más importantes de la actividad productiva, empleando además variables internas de las firmas.

Es importante exponer que el objetivo de este artículo es demostrar que existe una respuesta diferencial de las empresas, de los sectores más representativos de la economía colombiana, ante variaciones del entorno macroeconómi$\mathrm{co}$, analizando variables como producto 
interno bruto departamental ${ }^{1}$, inflación por ciudad, tasa de desempleo departamental, tasa de interés ${ }^{2}$ y variación del índice de tasa de cambio real ${ }^{3}$.

A pesar del pequeño stock de trabajos empíricos y/o teóricos, precedentes a éste, es importante mencionar a Sharpe (1981) y Schankerman (2002) quienes citados por Santoro y Gaffeo (2009), aceptan la importancia de los determinantes macroeconómicos en la probabilidad de supervivencia de las empresas. Siguiendo esta línea, Audretsch y Acs (1994), aseguran que la coyuntura de las variables macroeconómicas, influyen en el repunte de las firmas. Particularmente, Long (2010), propone que los ingresos operacionales de las empresas del sector construcción de Taiwán, dependen de fuerzas económicas y estatales de dicho país.

Igualmente, en Chile, Benavente y Külzer (2006), exponen que la tendencia desfavorable del producto interno bruto, tasa de desempleo, tasa de interés y tasa de cambio; además de factores como localización geográfica, edad, sector y competencia industrial, incidieron en la destrucción de empresas entre 1999 y 2006. Machin y Van Reenen (1993) afir- man que los shocks de la demanda británica durante 1980, afectaron el rendimiento empresarial de ese país, verificando esta afirmación, con variables macroeconómicas y factores temporales, sociales y políticos.

En Colombia, Ortiz (2005), plantea la existencia de ciclos en los cuales se establecen relaciones entre el entorno y el desempeño operativo de las empresas; fundamentando su conclusión en un análisis de las compañías manufactureras de Antioquia entre 1990 y 1995. A su vez, Aguilar (2003), expone que diferencias de tamaño y nivel de endeudamiento de las firmas colombianas, hacen que éstas reaccionen de manera diferencial ante las condiciones del entorno macroeconómico. Finalmente, Langebaek (2008: 2), realiza una investigación acerca de los determinantes del comportamiento de los ingresos operacionales de las empresas colombianas para los años 2005 y 2006; observando la existencia de escalas óptimas eficientes en las firmas.

A partir de estos referentes científicos sobre los determinantes de los ingresos operacionales empresariales, este trabajo explica mediante modelaciones econométricas con datos de panel, el im-

1 De acuerdo a con el Artículo 298 de la actual Constitución Política de Colombia, Departamento es una entidad territorial que goza de autonomía para la administración de los asuntos seccionales y la planificación y promoción del desarrollo económico y social dentro de su territorio en los términos establecidos por la Constitución y las leyes.

2 Tasa de interés (DTF) a 90 días. Es el resultado de promediar la tasa de captación de 90 días de los Certificados de Depósitos a Término (CDT's) de todos los intermediarios financieros, ponderados por el monto captado.

3 Índice de Tipo de Cambio Real Efectivo (ITCR), cuya fuente es el Fondo Monetario Internacional (FMI) y es calculado para el peso colombiano frente a 18 países miembros. 
pacto del entorno macroeconómico -variables antes citadas ${ }^{4}$, objetivos de este trabajo- y el de efecto variables propias de las firmas tales como activos, pasivos y edad; el desempeño operacional de las empresas de los sectores colombianos más representativos entre 1998 y 2009. Adicionalmente, se usan variables dicotómicas, relacionadas con la ubicación geográfica de las firmas ${ }^{5}$. La fuente de información de las variables macroeconómicas es el Departamento Administrativo Nacional de Estadísticas (DANE) y el Banco de la República. Las variables empresariales fueron tomadas de la Superintendencia de Sociedades de Colombia (Supersociedades).

Metodológicamente se emplearon datos en panel, para observar el movimiento de las unidades transversales en el tiempo lo que garantiza eficiencia en la estimación. El esquema de modelación seleccionado se especificó entre efectos fijos y efectos aleatorios de acuerdo a la Prueba de Hausman, para validar o rechazar la ortogonalidad de los efectos aleatorios. Los problemas de autocorre- lación y heterocedasticidad se detectaron con el Test de Wooldrige y la Prueba modificada de Wald; a su vez se empleó el método de Errores Estándar Corregidos para Panel (PCSE) ${ }^{6}$.

\section{Entorno macroeconómico: Colombia y sus regiones 1998-2009}

En este apartado es importante exponer, que Colombia atravesó una de las crisis económicas más fuertes de su historia durante los años 1998 y 1999, cuyo precedente comparativo, sólo fue la contracción de los años treinta. Los detonantes se atribuyen al sistema financiero, que a su vez afectó rubros importantes de la economía, en particular los sectores construcción, industria y comercio.

Pese a esto, el país, comenzó un proceso de recuperación desde el año 2000 , resultado de incrementos en la demanda interna y reactivación de los sectores más importantes de la economía nacional. Otro factor determinante en el reacondicionamiento de la economía fue

4 Producto interno bruto departamental, inflación por ciudad, tasa de desempleo departamental, tasa de interés y variación del índice de tasa de cambio real.

5 Sólo se utilizan las variables dicotómicas regionales en los modelos que se ajusten a efectos aleatorios, porque en efectos fijos, estas variables dummies provocan endogeneidad, al confundirse con los diferentes interceptos estimados para cada unidad transversal. Concretamente, los efectos fijos solamente permiten variables que cambian en el tiempo (Wooldridge, 2007: 515). El estimador de efectos fijos utiliza la variación "within" (intragrupos), por tanto los coeficientes de variables con poca variación "within" serán bastante imprecisos. Las variables dicotómicas representan la región Caribe, Pacífica y Orinoquía, la región Andina se emplea como categoría de comparación.

6 Beck y Katz (1995) reportaron que si la muestra es finita, estimaciones de Mínimos Cuadrados Ordinarios (OLS) con errores estándar corregidos para panel son más eficientes que los derivados de FGLS (Mínimos Cuadrados Generalizados Factibles) (Camacho García y Flamand, 2008: 283). 
Variables macroeconómicas e ingresos operacionales de las empresas colombianas... Yánez Contreras, Martha Alicia y Angulo Pico, Grace Margarita

el aumento de los niveles de inversión como resultado de mejores condiciones internas, tanto políticas como sociales.

\subsection{Producto interno bruto (PIB)}

Con respecto al producto interno bruto, la crisis de finales de los noventa, provocó que éste se redujera $-0,2 \%$ en 1998 y $-4,5 \%$, al cerrar el año 1999.

Bogotá, fue el ente territorial que más evidenció la contracción nacional, mostrando un descenso en su tasa de crecimiento $(-10,8 \%)$ en 1999. En 2005, esta ciudad experimentó su mayor variación del producto $(38,8 \%)$ aproximadamente. Tendencia parecida presentaron los departamentos de Antioquia y Valle, evidenciando sus mayores tasas de crecimiento en 2005. Durante los años restantes, el comportamiento de los productos departamentales fue uniforme, manteniéndose creciente (Tabla 1 y Gráfico 1). Es importante exponer que posterior a 2007 , se presentaron presiones de precios en los mercados internacionales, por lo que el Banco de la República tomó medidas de freno a nivel nacional, para desacelerar las compras de bienes de consumo y bienes externos (Fedesarrollo, 2008: 3). En 2008, se presentaron fenómenos de oferta y demanda que afectaron el producto, en el marco de la crisis financiera internacional que redujo las exportaciones y las remesas.

Estas circunstancias comenzaron a sentirse a nivel departamental en 2009, ya que las tasas de crecimiento de los productos fueron menores a $1 \%$, en la mayoría de los casos, reflejando la recesión mundial, la caída de la inversión, la disminución del recaudo del gobierno y el estancamiento general de la demanda agregada (Banco de la República, 2009: 24).

\subsection{Tasa de inflación}

Al ser técnicamente catalogada la caída de la actividad económica en 1999 como

\section{Tabla 1}

Tasa de crecimiento PIB principales departamentos 1999-2009

\begin{tabular}{cccccc}
\hline Años & Antioquia & Atlántico & Bogotá D. C. & Cundinamarca & Valle \\
\hline 1999 & $-2,5 \%$ & $-5,3 \%$ & $-10,8 \%$ & $-4,6 \%$ & $-3,6 \%$ \\
2000 & $5,7 \%$ & $3,7 \%$ & $3,7 \%$ & $7,4 \%$ & $1,1 \%$ \\
2001 & $-0,6 \%$ & $-0,5 \%$ & $1,3 \%$ & $11,2 \%$ & $2,9 \%$ \\
2002 & $4,9 \%$ & $1,9 \%$ & $4,3 \%$ & $-1,3 \%$ & $-0,3 \%$ \\
2003 & $4,8 \%$ & $6,4 \%$ & $3,3 \%$ & $3,4 \%$ & $1,5 \%$ \\
2004 & $4,7 \%$ & $8,3 \%$ & $6,9 \%$ & $1,0 \%$ & $4,0 \%$ \\
2005 & $16,4 \%$ & $7,2 \%$ & $38,8 \%$ & $23,6 \%$ & $17,7 \%$ \\
2006 & $7,5 \%$ & $5,9 \%$ & $7,2 \%$ & $5,9 \%$ & $8,7 \%$ \\
2007 & $4,1 \%$ & $14,4 \%$ & $12,6 \%$ & $0,9 \%$ & $6,2 \%$ \\
2008 & $2,4 \%$ & $1,9 \%$ & $3,3 \%$ & $5,7 \%$ & $3,3 \%$ \\
2009 & $0,5 \%$ & $0,8 \%$ & $1,3 \%$ & $0,1 \%$ & $0,2 \%$ \\
\hline
\end{tabular}

Fuente: Elaboración propia. 


\section{Gráfico 1 \\ PIB principales departamentos 1998-2009 (en millones de pesos)}

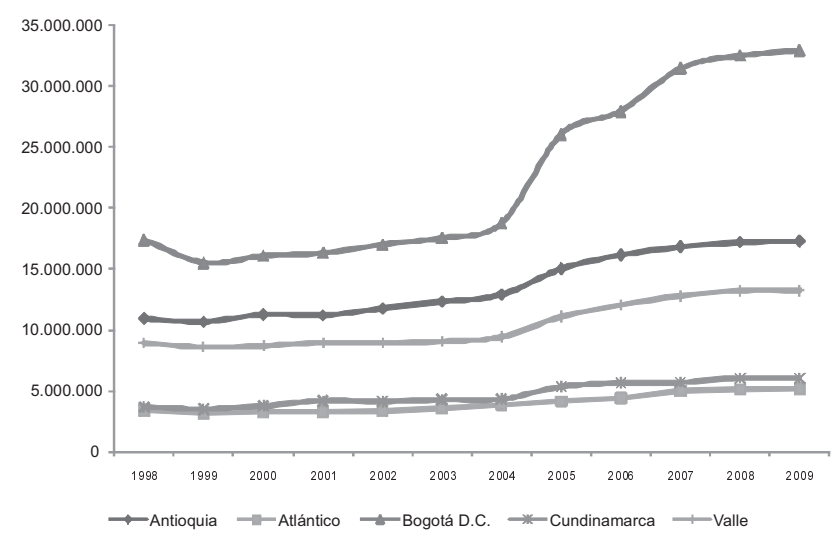

Fuente: Elaboración propia.

una recesión, la inflación se redujo por la disminución de la demanda de bienes y servicios (Gráfico 2). Posterior a esta situación recesiva, la tendencia de esta variable mostró un cambio estructural, debido al nuevo esquema de inflación objetivo que comenzó a adoptar la política monetaria del país. Pese a esto, en 2007 hubo presiones inflacionarias, por el incremento de los precios de las materias primas y alimentos en especial, además de problemas de oferta a causa del "fenómeno del niño", lo que llevó al incumplimiento de la meta en 2007 y 2008. Sin embargo 2009, fue un año con menos presiones inflacionarias, pero aún permanecían las consecuencias de la recesión mundial (Banco de la República, 2009: 16).

\subsection{Tasa de desempleo}

Esta variable llegó a su nivel más elevado en 1999, tanto a nivel nacional, como a nivel departamental. La principal causa de este aumento fue la desaceleración de la economía, debido a que las tasas de crecimiento del producto, fueron menores que la variación de la población económicamente activa, representando esto desempleo cíclico. No obstante, también se presentó acumulación de tasas de desempleo de períodos anteriores, por lo que simultáneamente hubo paro estructural.

Aunque el año 2000 mostró indicios de recuperación económica, el desempleo continuó en tasas muy elevadas en los principales departamentos del país. Sólo hasta el período 2002-2003, se presentó una reducción sostenida, que permaneció hasta 2007, año en que incrementaron las tasas de desocupación en la mayoría de departamentos (Gráfico 3), debido a que la crisis financiera condujo a la baja utilización de la capacidad productiva del país y por tanto a la destrucción de puestos de trabajo (Banco de la República, 2009: 30). 


\section{Gráfico 2 \\ Tasa de inflación principales áreas metropolitanas 1998-2009}

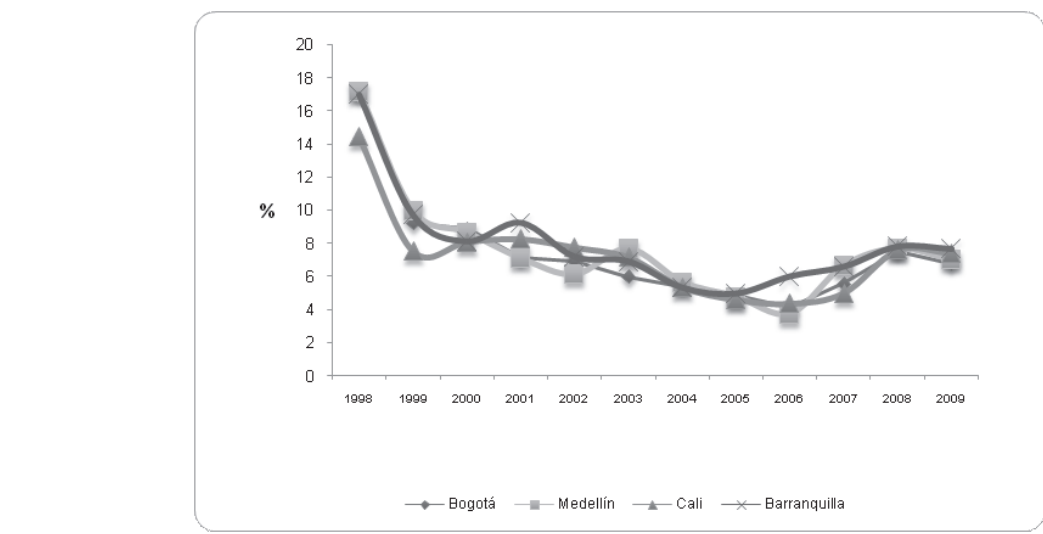

Fuente: Elaboración propia.

\section{Gráfico 3}

Tasa de desempleo principales departamentos 1998-2009

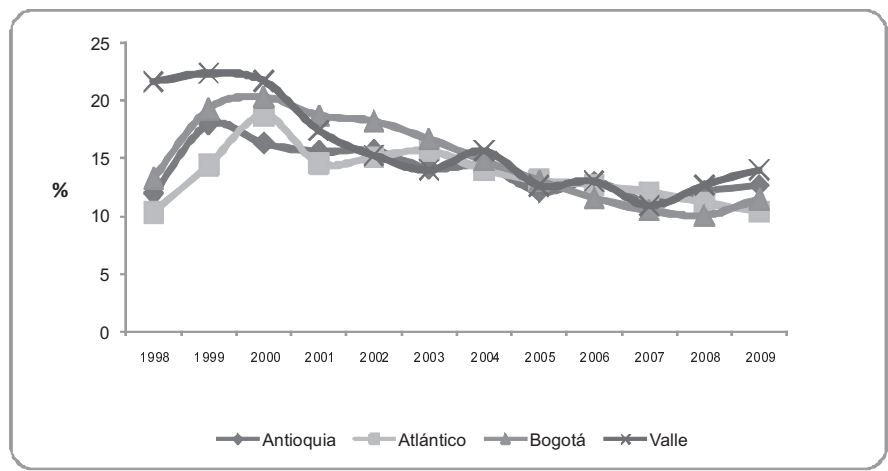

Fuente: Elaboración propia.

\subsection{Tasa de interés (DTF) y variación índice tasa de cambio real}

Estas dos variables se pueden fusionar porque tienen objetivos comunes. En 1999, el afán de la autoridad monetaria por mantener el tipo de cambio dentro de bandas cambiarias, llevó al aumento de la tasa de interés. Posteriormente, se adoptó un tipo de cambio flexible que ha permitido a la tasa de interés mostrar un cambio estructural en su tendencia. El fenómeno de expansión de la política monetaria, también propició la devaluación de la moneda colombiana a causa del exceso de dinero y la no entrada de capitales extranjeros. Sin embargo, entre 2002 y 2005 , se presentó una gran revaluación, que rompió su senda entre 2006 y 2007. Es de anotar que entre 2008 y 
2009, se presentó un fenómeno de aumento de las remesas, lo que contribuyó de manera decisiva a la valoración del peso colombiano (Gráfico 4) (Banco de la República, 2008: 39).

Una vez descrito el contexto macroeconómico que predominó en Colombia a nivel nacional y departamental entre 1998 y 2009 , es momento de analizar el comportamiento de los sectores representativos, expuestos a los escenarios económicos predominantes durante ese período.

\section{Principales variables financieras de los sectores representativos}

En este apartado se mostrará de manera breve la tendencia y los aspectos más importantes que marcaron la coyuntura de las empresas de los sectores energía; agropecuario; químico, construcción; comercio y otros servicios en Colombia, entre 1998 y 2009.
En términos generales el comportamiento de los ingresos operacionales fue consecuente con la coyuntura positiva que vivió la economía colombiana posterior a la crisis de 1999, resultado de la confianza y expectativas sobre su evolución en el mediano y largo plazo (Superintendencia de Sociedades de Colombia, 2004: 1).

Respecto al sector energía, es claro que Colombia es un país rico en recursos energéticos, situación que se estimuló aún más en la década de los noventa, por el descubrimiento de pozos petroleros; que significaron una mayor participación de este sector dentro del PIB nacional (Superintendencia de Sociedades de Colombia, 2007). Sin embargo es de anotar que el $62 \%$ de los ingresos sectoriales aproximadamente, se concentra en sólo cinco empresas Exxonmobil de Colombia S.A, DrummondLtd, Carbones del Cerrejón Limited, BP ExplorationCompany Colombia Limited y Hocol S.A.

De acuerdo con el Departamento Administrativo Nacional de Estadística

\section{Gráfico 4}

\section{Tasa de interés y variación índice tasa de cambio real 1998-2009}

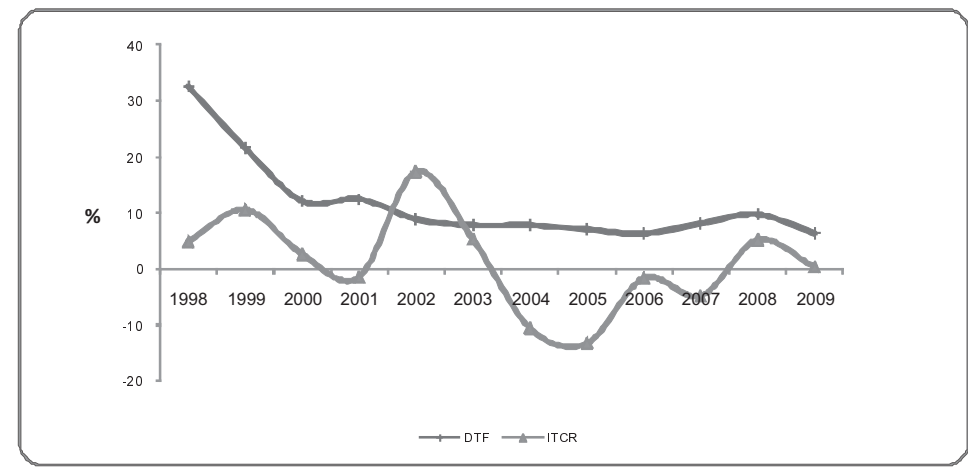

Fuente: Elaboración propia. 
(DANE), el crecimiento de la producción del sector ha estado en aumento sostenido desde 2003, después de la recuperación de la recesión de 1999 (Gráfico 5).

El sector agropecuario colombiano por su parte, es tradicional, cuyo comercio exterior ha estado representado básicamente por commodities, es decir, bienes primarios o materias primas sin ningún procesamiento o transformación. Los más exportados son: café, banano, flores y maíz (Superintendencia de Sociedades de Colombia, 2004: 2).

Del contexto macroeconómico, las variables que más afectan a este sector son las elevadas tasas de interés y la revaluación, porque crean un ambiente que desestimula la inversión. En este sentido, la apertura económica afectó negativamente al sector, porque lo expuso al fenómeno adverso de la tasa de cambio real, la reducción de precios internacionales y la mayor protección de los países industrializados (Rosas, 2007: 16).
Es importante destacar que el sector presentó su mejor tendencia a partir de 2007 (Gráfico 6), por el auge de los biocombustibles, mayor demanda de alimentos por parte de India y China; además de factores climáticos favorables (Arias, 2007).

Siguiendo con el sector químico, su dinamismo actual es resultado del incremento de las exportaciones hacia Venezuela, Ecuador y Perú (Gráfico 7). No obstante, existe gran dependencia a las materias primas importadas, lo que no le permite ser más competitivo internacionalmente (Cámaras de Usuarios de Zonas Francas. Asociación Nacional de Industriales, 2009).

En cuanto al sector construcción, éste experimenta ciclos de actividad mucho más acentuados que otras ramas productivas, demostrando la caída más pronunciada de los ingresos $(-38,3 \%)$ en 1999 (Gráfico 8), si se compara con los restantes sectores analizados en este trabajo. Es de anotar que los ciclos de

\section{Gráfico 5}

PIB sector energía 2002-2008

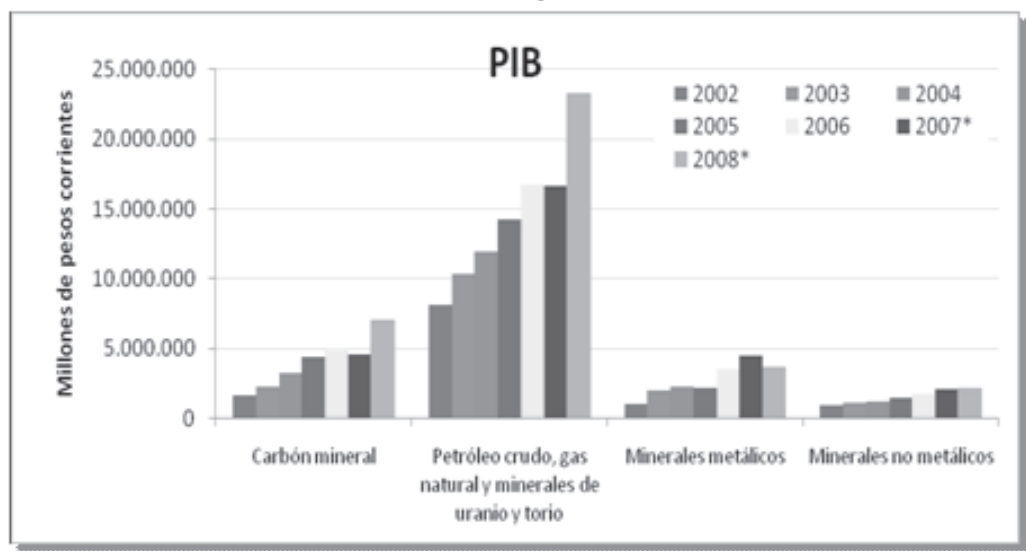

Fuente: Consejo Intergremial de Minería en Colombia (CIMCO) et al. 2009:11). 
esta industria, se presentan de forma muy pronunciada por lo que se espera una alta elasticidad de los ingresos del sector ante el vector de variables macroeconómicas. En los últimos años, al igual que el resto de la economía colombiana, el renglón construcción también presentó claras señales de desaceleración. Este menor dinamismo generó la expulsión de trabajadores vinculados a este rubro productivo, lo que sugiere evidentemente una relación negativa entre estas dos variables (Cámara Colombiana de la Construcción (CAMACOL), 2008: 17).

\section{Gráfico 6}

\section{Tasa de crecimiento de los ingresos operacionales sector} agropecuario 1999-2009

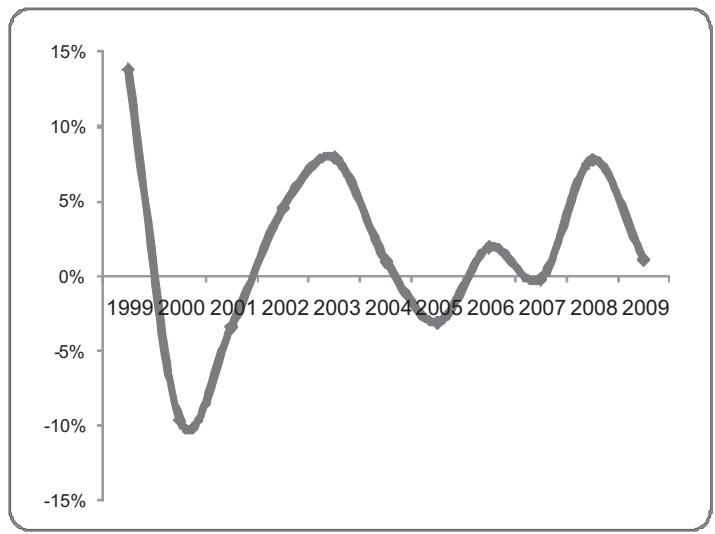

Fuente: Elaboración propia.

\section{Gráfico 7}

Sector químico 1998-2009 (ingresos-activos-pasivos) (valores en millones de peso)

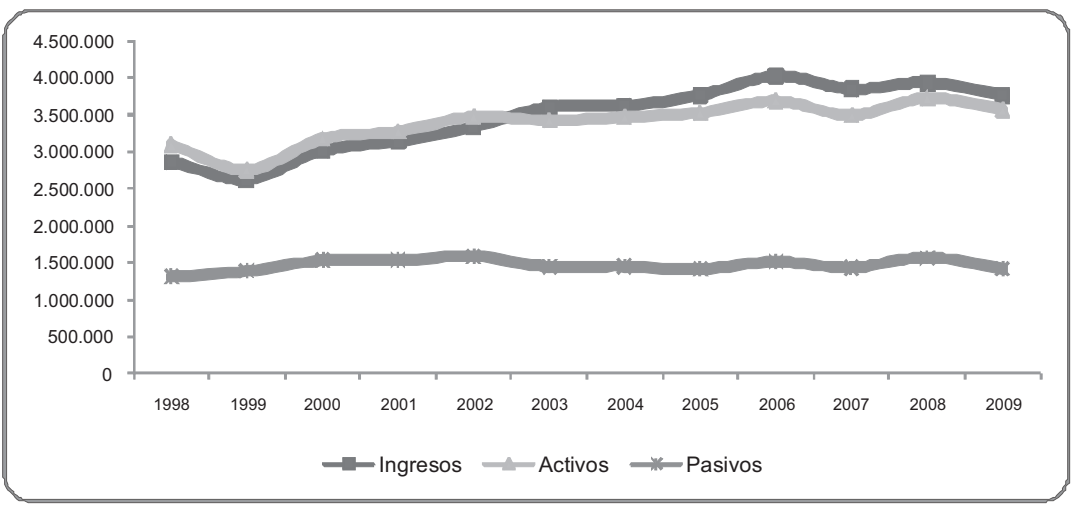

Fuente: Elaboración propia. 


\section{Gráfico 8 \\ Tasa de crecimiento de los ingresos operacionales sector construcción 1999-2009}

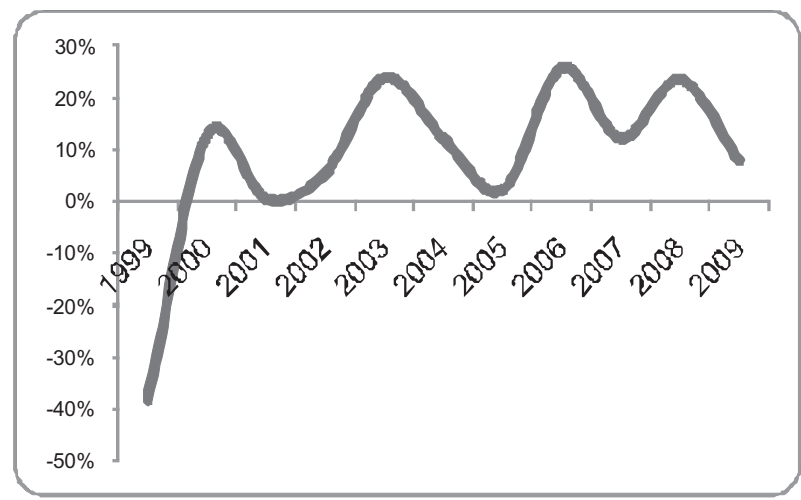

Fuente: Elaboración propia.

En cuanto al sector comercio, en los últimos tiempos se ha presentado una consolidación de las empresas dedicadas a esta actividad, por la presencia de grandes multinacionales. Además el proceso de apertura económica de principios de los noventa, propició las condiciones para su expansión, la cual se vio debilitada por la recesión de 1999. A partir de ese período la tendencia operacional del sector se mantuvo creciente, con tasas de crecimiento mayores a $7 \%$, excepto entre 2007 y 2009, debido a los efectos de la crisis financiera internacional (Gráfico 9).

Finalmente el sector denominado "otros servicios", representa fielmente la terciarización de la economía producto del cambio de la estructura productiva del país, desde mediados del siglo XX. Esto ha sido jalonado por los sectores comunicaciones, transporte, servicio a las empresas y servicio al gobierno Moncayo y Garza, 2005 citado por Bonet (2006: 1). Este último autor, citando a Weller (2004) admite que los avances tecnológicos de los últimos tiempos, hacen que los servicios sean cada vez, más intensivos en capital; situación coherente con la importancia que tienen los activos dentro del sector, tal y como se refleja en el Gráfico 10, que también muestra que operacionalmente sólo se vio en riesgo en 1999, durante la recesión.

Este apartado dejó claro que los sectores representativos de la economía colombiana analizados, entre 1998 y 2009 , se comportaron guardando correspondencia con lo sucedido a nivel macroeconómico, presentando respuestas procíclicas ante las variaciones de los agregados, pero diferencias de acuerdo a sus características internas.

En la siguiente sección, se presenta la cuantificación del impacto del entorno macroeconómico departamental y nacional de Colombia, sobre los ingresos operacionales de los sectores representativos, incluyendo variables internas de las empresas, en busca de obtener un mejor ajuste en la modelación. 


\section{Gráfico 9 \\ Sector comercio 1998-2009 (ingresos- activos-pasivos) \\ (valores en millones de pesos)}

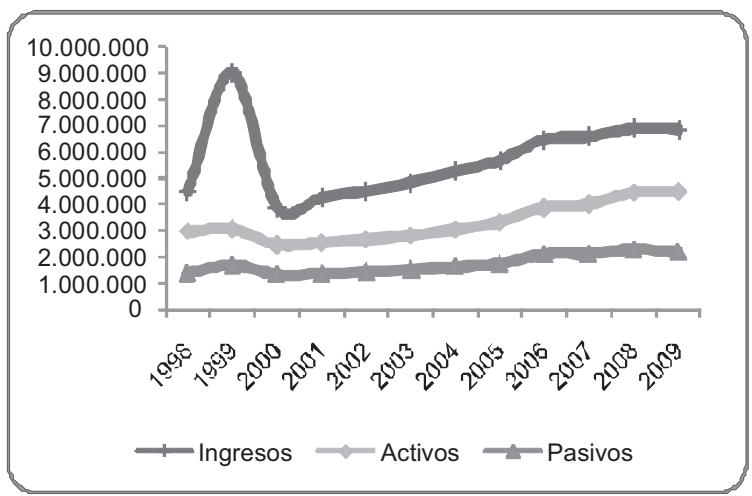

Fuente: Elaboración propia.

Gráfico 10

Sector otros servicios 1998-2009 (ingresos- activos-pasivos)

(valores en millones de pesos)

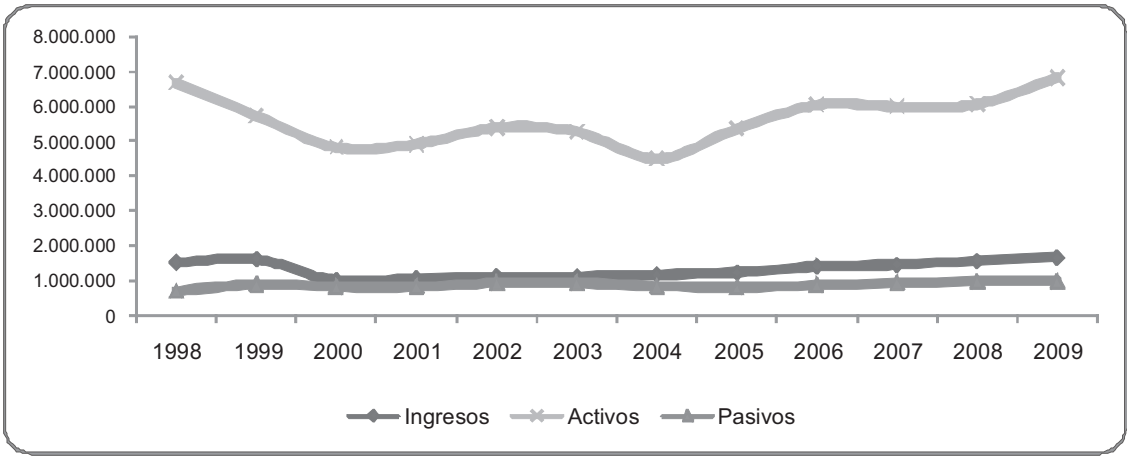

Fuente: Elaboración propia.

4. Modelos de impacto macroeconómico: ingresos operacionales, empresas de sectores representativos 1998-2009

Después de exponer el entorno macroeconómico colombiano y las principales tendencias de los sectores repre- sentativos del aparato productivo del país, durante los doce años que abarca la serie temporal de esta investigación; se pasa a la estimación de los modelos econométricos sectoriales, mediante la metodología de datos de panel.

En primer término es necesario realizar una breve descripción de la base de datos empresarial empleada para la 
modelación, la cual contó 1325 empresas pertenecientes a los sectores más representativos de la economía colombiana. Es de anotar que estas empresas reportaron sus estados financieros ante la Superintendencia de Sociedades de Colombia, durante el período de estudio. En total se seleccionaron los seis sectores considerados los más representativos de la economía del país, por el aporte de sus ingresos operacionales al producto interno bruto. Entre ellos: energía (extracción de carbón, petróleo y otros derivados), agropecuario, químico, construcción, comercio y otros servicios (Gráfico 11). En el anterior apartado se mostró la tendencia financiera y comportamiento de los mismos.

Las cifras sectoriales demuestran la terciarización de la economía colombiana, porque aproximadamente el $58 \%$ de las firmas analizadas (Gráfico 11), corresponden a los sectores comercio y otros servicios.

En cuanto a la ubicación geográfica, se observa que los departamentos más representativos de acuerdo al número de empresas son: Bogotá con $54 \%$, Antioquia y Valle con $13,4 \%$ y $12,8 \%$, respectivamente (Gráfico 12). Esto eviden-

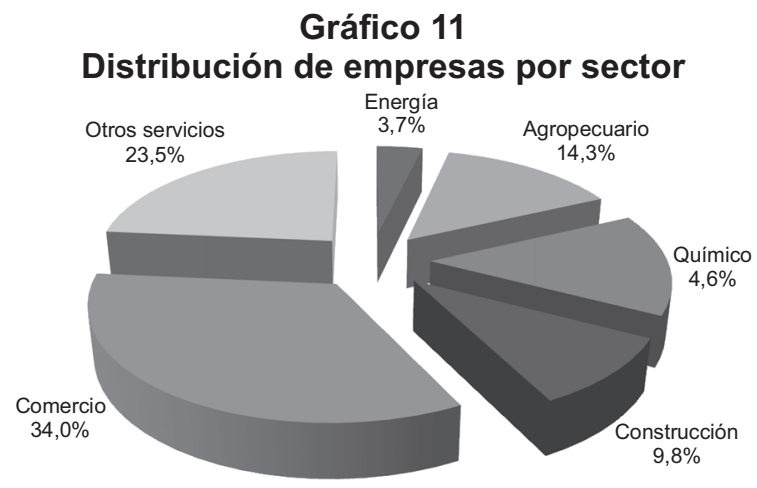

Fuente: Elaboración propia.

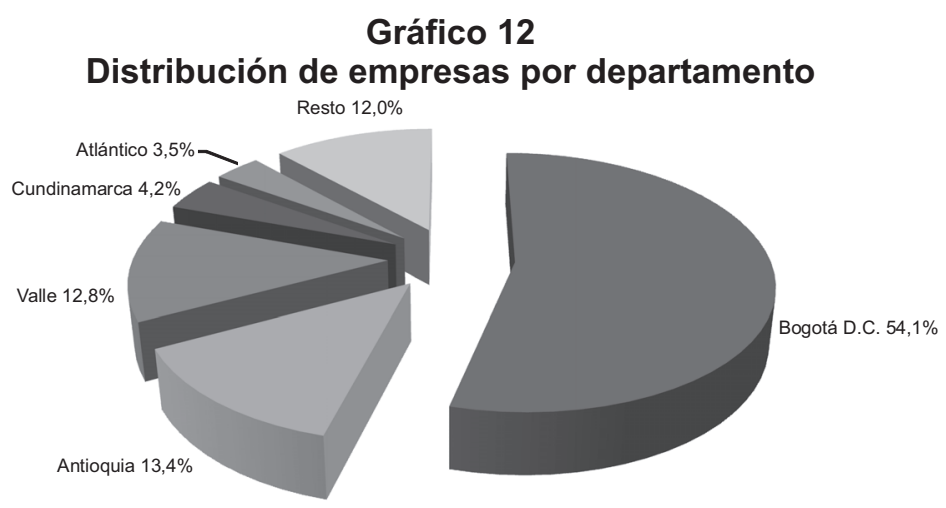

Fuente: Elaboración propia. 
cia la importancia de las regiones Andina y Pacífica (Gráfico 13).

Econométricamente se estimaron seis paneles sectoriales. Estos paneles son balanceados porque las empresas que se incluyeron, permanecieron durante todo el período, garantizando así, que son lo suficientemente productivas como para mantenerse dentro de la industria (López, 2006: 54).

Es importante destacar la importancia teórica de cada una de las variables empleadas en las modelaciones sectoriales, así como las técnicas econométricas empleadas y el análisis de las fuentes de información.

La variable dependiente está representada por los ingresos operacionales de las empresas de cada uno de los sectores representativos, cuya fuente de información es la superintendencia de sociedades de Colombia.

Las variables explicativas o independientes son: edad de las empresas; tamaño (activos); nivel de endeudamiento (pasivos); variables dicotómicas regionales y un vector de variables macroeconómicas (Cuadro 1).
Con respecto al modelo teórico, a partir de la división de empresas por sector, se establece el siguiente:

$$
\begin{aligned}
\text { Iny }= & \beta_{1}+\beta_{2} \operatorname{lna}+\beta_{3} \operatorname{lnp}+\beta_{4} \mathrm{edad}+\beta_{5} \mathrm{dc}+ \\
& \beta_{6} \mathrm{dp}+\beta_{7} \mathrm{do}{ }^{11}+\beta_{9} \operatorname{lnpib}-\beta_{10} \mathrm{i}-\beta_{11} \mathrm{~d}- \\
& \beta_{12} \mathrm{r}+\beta_{13} \text { Vartc }+\varepsilon
\end{aligned}
$$

donde

In: logaritmo neperiano.

$y$ : ingresos operacionales

(millones de pesos).

a: activos totales (millones de pesos).

$\mathrm{p}$ : pasivos totales (millones de pesos).

edad: número de años de funcionamiento de la firma.

dc: variable dicotómica que representa la región caribe. 1 si pertenece a la región, 0 en otro caso.

$\mathrm{dp}$ : variable dicotómica que representa la región pacífica. 1 si pertenece a la región, 0 en otro caso.

pib: producto interno bruto por departamento (millones de pesos).

i: inflación por área metropolitana (\%).

$\mathrm{d}$ : tasa de desempleo por departamento (\%).

$r$ : tasa de interés (DTF) (\%).

vartc: variación del índice de tasa de cambio real $(\%)$.

\section{Gráfico 13}

Distribución de empresas por región

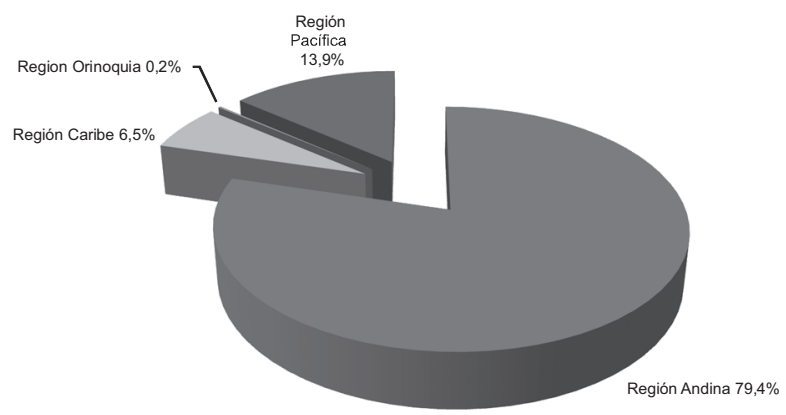

Fuente: Elaboración propia. 
Variables macroeconómicas e ingresos operacionales de las empresas colombianas... Yánez Contreras, Martha Alicia y Angulo Pico, Grace Margarita

\section{Cuadro 1}

Operacionalización de las variables

\begin{tabular}{|c|c|c|c|}
\hline Variable & Naturaleza & Indicador & Fuente \\
\hline Ingresos Operacionales & Dependiente & Millones de pesos & $\begin{array}{l}\text { Superintendencia } \\
\text { de Sociedades } \\
\text { de Colombia } \\
\text { (Supersociedades) }\end{array}$ \\
\hline Edad de la Empresa & Independiente & Años de funcionamiento & Supersociedades \\
\hline Tamaño de la Empresa & Independiente & $\begin{array}{l}\text { Activos Totales en } \\
\text { Millones de Pesos }\end{array}$ & Supersociedades \\
\hline Endeudamiento & Independiente & $\begin{array}{l}\text { Pasivos Totales en } \\
\text { Millones de Pesos }\end{array}$ & Supersociedades \\
\hline $\begin{array}{l}\text { Producto Interno Bruto } \\
\text { Departamental }\end{array}$ & Independiente & Millones de pesos & $\begin{array}{c}\text { Departamento } \\
\text { Administrativo Nacional } \\
\text { de Estadística (DANE) }\end{array}$ \\
\hline $\begin{array}{l}\text { Inflación por Área } \\
\text { Metropolitana }\end{array}$ & Independiente & Variación Anual del IPC & (DANE) \\
\hline $\begin{array}{l}\text { Tasa de Desempleo } \\
\text { Departamental }\end{array}$ & Independiente & Tasa de Desempleo (\%) & (DANE) \\
\hline Tasa de Interés & Independiente & $\mathrm{DTF}^{7}$ a 90 días (\%) & Banco de la República \\
\hline $\begin{array}{c}\text { Índice de Tasa de } \\
\text { Cambio Real }\end{array}$ & Independiente & $\begin{array}{c}\text { Índice de Tasa de } \\
\text { Cambio Real, base } \\
1994^{8}\end{array}$ & Banco de la República \\
\hline
\end{tabular}

Fuente: Elaboración propia.

En tal sentido, las variables seleccionadas quedarían:

a. (y): ingresos operacionales. Representa la dinámica productiva y comercial de las firmas.

b. (a): activos totales. El tamaño de las firmas, es determinante del crecimiento de sus ventas, de acuerdo a la teoría neoclásica de escala mínima eficiente y las teorías que afirman que entre más grande sea la empresa, mayor es su poder negociación, su potencial para ser rentables y poseen mayor esfuerzo innovador (Schumpeter, 1934) en (Huerta et al., 2010: 214).

c. (p): pasivos: Se espera una relación inversa entre pasivos e ingresos ope-

7 Es el resultado de promediar la tasa de captación de 90 días de los Certificados de Depósitos a Término (CDT's) de todos los intermediarios financieros, ponderados por el monto captado.

8 Índice de Tipo de Cambio Real Efectivo ITCR:, cuya fuente es el Fondo Monetario Internacional (FMI) y es calculado para el peso colombiano frente a 18 países miembros. 
racionales, debido a que las firmas más endeudadas tienden a descuidar sus resultados operacionales. Sin embargo, también es factible que un elevado nivel de deuda discipline a los administradores (Jensen, 1986), lo que sería coherente con un signo positivo de esta variable.

d. (edad): años de funcionamiento de la empresa. Langebaek (2008: 11), afirma que la mayor edad de una empresa puede ser indicio de madurez. Esta madurez, puede llevar a mejores relaciones con clientes y demás usuarios de las compañías. El autor también expone que el signo negativo de esta variable puede ser explicado porque en las empresas jóvenes, el gerente - administrador es el mismo fundador, quien es más disciplinado e imprime más voluntad de trabajo.

e. $(\mathrm{dc}, \mathrm{dp}, \mathrm{do})^{9}$. Variables dicotómicas que representan tres regiones geográficas de Colombia (región Caribe, región Pacífica y región Orinoquía), tomando la región Andina como categoría de comparación. Alfred Marshall, citado por Langebaek (2008: 3) afirma que la ubicación geográfica puede contribuir a mejorar la competitividad em- presarial. Scitovsky (1954) afirmó que la localización de las firmas puede generar economías pecuniarias por la abundancia de capital o trabajo calificado. A su vez, el territorio da pie a dinámicas económicas y sociales (Cardona y Cano, 2005). Sin embargo, asumen la existencia de disparidades que desembocan en regiones ganadoras y perdedoras. En el caso de esta investigación, a priori se tiene conocimiento que la región andina colombiana abarca gran cantidad de empresas y concentra la mayor parte de la economía del país.

f. (pib): producto interno bruto departamental. Representa la renta de cada departamento donde se encuentre ubicada la firma, es decir la capacidad de compra de los agentes económicos para adquirir bienes y servicios de las empresas. Por tanto, un incremento del pib, en teoría, debería aumentar los ingresos operacionales de las empresas, debido al incremento subyacente del poder de compra.

g. (i): inflación anual por área metropolitana ${ }^{10}$. Una variación positiva de la tasa de inflación, ocasiona pérdida de poder adquisitivo, y por tanto debería

9 Para esta investigación los departamentos de la región Andina son: Antioquia, Bogotá D.C., Boyacá, Caldas, Cundinamarca, Huila, Norte de Santander, Quindío, Risaralda, Santander y Tolima. De la región Caribe: Atlántico, Bolívar, César, Córdoba, Magdalena, San Andrés Providencia y Sucre. De la región Pacífica: Cauca, Valle y Nariño. De la región Orinoquía: Meta.

10 Las áreas metropolitanas que tiene en cuenta el Departamento Administrativo Nacional de Estadística son: Bogotá, Medellín, Cali, Barranquilla, Bucaramanga, Manizales, Pasto, Pereira, Cúcuta, Montería, Neiva, Cartagena y Villavicencio. Por esta razón se clasificaron las empresas de cada sector de acuerdo al departamento al que pertenecen y se asignó la inflación de la ciudad capital de cada uno de ellos. Las empresas cuyas capitales de departamento no se hallaban entre las 13 áreas metropolitanas, fueron expuestas a la inflación nacional. 
causar reducción del crecimiento de los ingresos operacionales.

h. (d): tasa de desempleo departamental. Entre mayor sea la tasa de desempleo, menores son las posibilidades de compra de bienes por parte de los agentes económicos, por lo que deben reducirse los ingresos operacionales de las empresas.

i. (r): tasa de interés DTF a 90 días. Teóricamente, al incrementar la tasa de interés de captación, aumenta la rentabilidad del ahorro y por tanto se reduce la cantidad de la renta destinada a la compra de bienes y servicios por parte de los agentes económicos. Esto debe reducir los ingresos operacionales de las empresas. Igualmente incrementos en la tasa de interés de colocación, aumentan el costo del dinero y por tanto reducen la inversión y los ingresos corporativos.

j. (vartc): variación índice de tasa de cambio real. Una variación positiva del índice de tasa de cambio real, debería ocasionar una variación de los ingresos operacionales en la misma dirección, debido al incremento previo de las exportaciones. La revaluación (variación negativa), por su parte, beneficia la importación de maquinaria y por tanto, también mejora los ingresos de las empresas.

Técnicamente, las variables en unidades monetarias como ingresos operacionales, activos, pasivos y producto interno bruto departamental (PIB) se manejaron con logartimo neperiano, para disminuir la variabilidad de los datos y poder reflejar cambios porcentuales estimando elasticidades. La variable edad de las firmas, se trabajó como años de funcionamiento y las variables porcentuales como tasa de inflación, tasa de desempleo y variación del índice de tasa de cambio real; permanecieron en su unidad original.

Al aplicar las pruebas correspondientes; resultó que los sectores energía y construcción debían ser estimados utilizando efectos aleatorios, bajo el argumento de que las empresas de estos sectores, tienen intercepto común y un componente aleatorio que se traslada al término estocástico de los modelos. Es importante argumentar que los sectores energía y construcción son excepcionales por considerarse locomotoras de la economía colombiana. Además una explicación a la presencia efectos aleatorios, es que los efectos inobservables entre las firmas de estos sectores, deben girar en torno a un valor medio. Adicionalmente, el sector energía presenta una estructura oligopólica, estructura que a pesar de sólo pertenecer a cinco empresas $^{11}$, es suficiente para dominar la totalidad de la industria y condicionar la tendencia de las firmas restantes.

Bajo este contexto, en estos sectores, debido a la presencia de efectos aleatorios, las variables dicotómicas regionales toman importancia explícita.

Los restantes sectores se estimaron siguiendo el esquema de efectos fijos, con variables dicotómicas de inter-

Ver en el apartado anterior la caracterización del sector energía. 
cepto diferentes para cada una de las unidades transversales. Éstas se interpretan como efectos inobservables propios de cada firma, relacionados con patrones de administración y con regionales, culturales, sociales, entre otros. En la teoría empresarial, las firmas se distinguen por sus patrones de administración, esquemas de propiedad y estrategias de crecimiento; siendo esta última de gran importancia para los resultados operativos, ya que de una estrategia de diversificación y/o especialización, pueden surgir altos o bajos niveles de desempeño operacional (Huerta et al., 2008). Todas estas variables están implícitas en el componente inobservable del estimador de efectos fijos.

Dentro de los modelos estimados con efectos aleatorios, explícitamente se plantea la existencia de factores regionales determinados por la ubicación geográfica de las empresas, las cuales desarrollan relaciones territoriales. De acuerdo a la distribución y el desempeño de las variables macroeconómicas departamentales, se evidenció que Bogotá y Antioquia son los departamentos líderes en cantidad de firmas y en comportamiento de su economía; por esta razón, se tomó como categoría de comparación la región andina en los modelos de los sectores energía y construcción.

En la Tabla 3 se presenta el resumen de las estimaciones de las distintas variables explicativas para las empresas de los sectores estudiados.

Debido a que el objeto de estudio de esta investigación es determinar el impacto del entorno macroeconómico en los ingresos operacionales de las firmas, es importante dividir las variables agregadas, en variables de política monetaria y de mercado de bienes. Las primeras, tasa de interés, variación del índice de tasa de cambio real y tasa de inflación ${ }^{12}$, y las segundas logaritmo neperiano del PIB y tasa de desempleo. Del primer grupo de variables, resultaron significativas: la tasa de interés $(r)$ y la variación del índice de tasa de cambio real (vartc) en los sectores químico y otros servicios. De esta manera se demuestra la importancia del sector externo en el desempeño de los ingresos de ambos sectores. En el sector químico particularmente, el signo de la relación de los ingresos con la tasa de cambio se explica por la gran dependencia del sector a las materias primas importadas. A su vez el signo del coefi-

12 Es claro que en la presentación del modelo IS-LM se especifican los mercados de mercancías, dinero y activos financieros, incluyendo también el mercado de trabajo, a través de la hipótesis de rendimientos marginales constantes del factor trabajo (Lizarazu, 2006). También es importante recordar que la estabilidad macroeconómica o equilibrio macroeconómico, implica el cumplimiento de objetivos de estabilidad de precios, pleno empleo, crecimiento económico, equilibrio en balanza de pagos y redistribución del ingreso (León, 2007: 3). León (2007: 33) también expone que Friedman consideraba que las variables monetarias influían sobre las reales de manera retardada, por ejemplo un proceso desinflacionario, implica pérdidas de empleo y producción en el corto plazo, pero con el paso del tiempo la economía retorna a su tasa natural de desempleo y producción. 
Tabla 3

Impacto ${ }^{13}$ macroeconómico en ingresos operacionales de las empresas colombianas. Sectores representativos 1998-2009

\begin{tabular}{lcccccc}
\hline Variables & Energía & $\begin{array}{c}\text { Agrope- } \\
\text { cuario }\end{array}$ & Químico & $\begin{array}{c}\text { Cons- } \\
\text { trucción }\end{array}$ & $\begin{array}{c}\text { Comercio } \\
\text { Otros } \\
\text { servicios }\end{array}$ \\
\hline Constante & $3,36^{* 14}$ & $7,95^{*}$ & $4,71^{*}$ & $3,12^{*}$ & $4,53^{*}$ & 1,08 \\
In activos (Ina) & $0,63^{*}$ & $0,37^{*}$ & $0,36^{*}$ & $0,37^{*}$ & $0,427^{*}$ & $0,54^{*}$ \\
In pasivos(Inp) & $0,25^{*}$ & 0,031 & $0,17^{*}$ & $0,27^{*}$ & $0,175^{*}$ & 0,214 \\
edad(edad) & 0,000 & $-0,01^{*}$ & $-0,003$ & $0,01^{*}$ & 0,001 & $0,03^{*}$ \\
región caribe (dc) & $-0,86^{*}$ & & & $-0,053$ & & \\
Región pacífica(dp) & 0,140 & & & 0,019 & & \\
Variación porcentual del pib & & & & & & \\
departamental (Inpib) & $-0,093$ & $-0,12^{*}$ & $-0,033$ & $-0,050$ & 0,037 & 0,105 \\
tasa de inflación por ciudad (i) & $-0,017$ & $-0,02^{*}$ & 0,038 & 0,026 & 0,009 & $-0,016$ \\
tasa de desempleo & & & & & & \\
departamental (d) & $-0,03^{*}$ & $-0,002$ & $-0,051$ & $-0,02^{*}$ & $-0,008$ & 0,009 \\
tasa de interés (r) & $-0,005$ & 0,006 & $-0,007^{*}$ & $-0,001$ & 0,004 & $0,02^{*}$ \\
variación índice de tasa & & & & & & \\
de cambio real (vartc) & 0,001 & 0,002 & $-0,003^{*}$ & $-0,004$ & $-0,001$ & $0,004^{*}$ \\
$R^{2}$ & 0,785 & 0,855 & 0,885 & 0,524 & 0,840 & 0,769 \\
Probchi2 Prueba de Hausman & 0,722 & 0,0000 & 0,0000 & 0,308 & 0,0000 & 0,0000 \\
\hline Fuen
\end{tabular}

Fuente: Elaboración propia.

ciente de tasa de interés se explica por el efecto expulsión que ejercen las alzas del costo del dinero sobre la inversión, variable necesaria, en un sector transformador de insumos. Particularmente, el subsector plástico, presenta un marcado déficit comercial por el alto volumen de sus importaciones de maquinaria, en comparación con las exportaciones de productos, lo que explica la necesidad de mantener bajas tasas de cambio (Cámara de Usuarios de Zonas Francas, 2008).

Respecto al sector otros servicios, su importancia con el comercio internacional radica en que los acuerdos comerciales de Colombia, además de buscar la liberación de los intercambios, intentan propiciar la regulación no discriminatoria para la inversión en este sector (Banco de la República, 2006: 2), inversión que se-

13 El método de estimación es panel balanceado por efectos fijos o aleatorios, de acuerdo a la validación de Hausman. Los errores estándar son robustos para heterocedasticidad y autocorrelación, incluyendo un término autorregresivo de primer orden AR (1).

14 *Significancia al nivel de 0,05. 
ría desestimulada con elevadas tasas de interés, dentro del país. Adicionalmente, en tiempos recientes, los servicios han sido identificados como un rubro potencial de exportación de Colombia, por las posibles ventajas que genera la mano de obra calificada, valorada más barata internamente respecto a otros países (Banco de la República, 2006: 5).

Otra variable considerada de carácter monetario es la tasa de inflación, la cual resultó concluyente y con signo esperado en el sector agropecuario, mostrando que elevados niveles de precios, perjudican la competitividad y por tanto el comportamiento operacional del sector.

Pasando a las variables denominadas de mercado de bienes (logaritmo neperiano del PIB y tasa de desempleo). En primera instancia, el logaritmo neperiano del PIB sólo resultó significativo y moderadamente importante en el sector agropecuario, aunque no con el signo esperado en teoría $(-0,12)$. La explicación puede estar en que el auge de los sectores intangibles, ha dejado de lado el sector agropecuario a nivel de demanda agregada, hecho que data desde el advenimiento de la apertura económica y la consecuente poca competitividad que existe en el sector, evidenciando escasez de modelos de organización empresarial que vayan más allá de las concentraciones productivas (Rugeles Chacón y Francois Jolly, 2006).

Pese a lo sucedido en el sector agropecuario, con la significancia hacia la variación del PIB departamental; es la tasa de desempleo, la variable del mercado de bienes, más determinante en los ingresos operacionales de las empresas específicamente de los sectores energía y construcción, mostrando que incremen- tos de un (1) punto porcentual en la tasa de desempleo departamental, afectan negativamente el desempeño operativo de los mismos en $-0,03$ y $-0,02$ puntos porcentuales respectivamente. La explicación para el sector energía, puede radicar en la importancia de la demanda interna del mismo, porque el Plan Energético Nacional, sienta las bases del aseguramiento de la disponibilidad y el pleno abastecimiento de los recursos energéticos para atender la demanda nacional, maximizar la cobertura y garantizar el desarrollo social (Corredor, 2008). Para el sector construcción, un incremento en la tasa de desempleo, según Saldarriaga (2006), desacelera su desempeño, por lo que es lógico esperar, reducción los ingresos operacionales de sus compañías.

Es de anotar que el sector comercio fue el único del total analizado, donde las variables macroeconómicas, resultaron no significativas. No obstante, el estadístico $z$ del logaritmo neperiano del PIB y la variación del índice de tasa de cambio en dicho sector, quedaron cercanos a la zona de significancia, con coeficiente positivo y negativo respectivamente, por lo que los ingresos operacionales de las empresas comerciales, son ligeramente procíclicos a la actividad agregada departamental, y dependientes al sector externo. Sin embargo, cuantitativamente se demuestra que los resultados operativos de las empresas del sector, son más la respuesta a la tendencia de sus activos y pasivos.

A modo de resumen, de acuerdo a las estimaciones, es claro que las variables propias de la estructura empresarial: activos y pasivos, son significativas en la mayoría de sectores, mostrando los ingresos operacionales, signos positivos $y$ 
altas elasticidades, especialmente respecto a los activos. Referente a los pasivos, la relación que evidencia esta variable financiera con los ingresos, es positiva, presentándose la mayor elasticidad en los sectores construcción y energía. De esta manera se verifica lo expuesto por Jensen (1986), quien afirma que un elevado nivel de deuda, puede disciplinar a los administradores y conducir la firma a mayores ingresos operativos. Con relación a la edad de las compañías, ésta resultó negativa en los sectores, agropecuario y construcción, demostrando lo expuesto por Langebaek (2008: 4), respecto a que las empresas más jóvenes son administradas por sus dueños, esperando más compromiso por parte de los mismos y por tanto mayores ingresos operacionales. Este autor también expone argumentos con relación al signo positivo de la edad que en este caso se presenta en el sector otros servicios, porque las empresas más antiguas, son más maduras y obtienen mayores ingresos.

Retornando a los modelos estimados con efectos aleatorios (sectores energía y construcción), las variables regionales sólo resultaron significativas en el primero, verificando la importancia es- perada de la región andina, sobre la Caribe (dc). En el sector construcción, el efecto regional no resulta concluyente, por lo que las diferencias entre las firmas de este gremio se califican como puramente estocásticas.

Los coeficientes autónomos en los sectores con estimador de efectos fijos, se interpretan como la variación porcentual (logaritmo neperiano) de los ingresos operacionales, de la empresa que se toma como base en cada sector. En este caso es la primera empresa que aparece en las bases de datos sectoriales, organizadas por orden alfabético. Debe ser claro, que las restantes compañías tienen coeficientes autónomos diferentes, demostrando que sus ingresos operacionales varían de manera independiente de acuerdo a efectos inobservables particulares de cada una de ellas; ya sea, relacionados con el modelo administración, entorno de trabajo, cualificación de sus empleados, infraestructura, entre otros. Por tanto se obtienen diferenciales para los interceptos de las empresas. Las compañías con las mayores variaciones porcentuales de ingresos operacionales, comparadas con la variación porcentual de la primera empresa de cada sector son (Tabla 4):

\section{Tabla 4}

Mayores variaciones de ingresos operacionales de las empresas colombianas. Sectores representativos 1998-2009

\begin{tabular}{|c|c|c|c|c|}
\hline Empresa & Agropecuario & Químico & Comercio & Servicios \\
\hline Avidesa Mac Pollo S.A. & 10,05 & & & \\
\hline Pimpollo S.A. & 9,40 & & & \\
\hline Alimentos Friko S.A. & 9,33 & & & \\
\hline C I Unión de Bananeros de Urabá S.A. & 9,23 & & & \\
\hline Pollo Fiesta S.A. en Concordato & 9,10 & & & \\
\hline
\end{tabular}


Tabla 4 (Continuación)

\begin{tabular}{|c|c|c|c|c|}
\hline Empresa & Agropecuario & Químico & Comercio & Servicios \\
\hline Industrias Puropollo S.A & 8,97 & & & \\
\hline Pollosan S.A & 8,96 & & & \\
\hline Boehringer Ingelheim S.A & & 6,69 & & \\
\hline Abbott Laboratories de Colombia S.A. & & 6,51 & & \\
\hline Goodyear de Colombia S.A & & 6,44 & & \\
\hline Cristalería Peldar S.A & & 6,35 & & \\
\hline Compañía Iberoamericana de Plásticos S.A & & 6,35 & & \\
\hline Dupont de Colombia S.A. & & 6,35 & & \\
\hline Belstar S.A. & & 6,32 & & \\
\hline Bayer S.A. & & 6,32 & & \\
\hline Laboratorios Baxter S.A. & & 6,31 & & \\
\hline Novartis de Colombia S.A & & 6,31 & & \\
\hline Distribuidora Colombina Ltda. & & & 4,61 & \\
\hline A Laumayer y Compañía S.A & & & 4,60 & \\
\hline $\begin{array}{l}\text { Herramientas Suspensiones } \\
\text { y Combustibles S. A }\end{array}$ & & & 4,60 & \\
\hline Grandes superficies de Colombia S.A & & & 4,55 & \\
\hline 3m Colombia S.A & & & 4,54 & \\
\hline Activos S.A. & & & & 2,84 \\
\hline Ahora S.A. & & & & 2,38 \\
\hline Acciones y Servicios S.A. & & & & 2,36 \\
\hline Adecco Colombia S.A. & & & & 2,27 \\
\hline Assenda S.A. & & & & 2,25 \\
\hline Acción S.A. & & & & 2,23 \\
\hline Ayuda Integral S.A. & & & & 2,11 \\
\hline
\end{tabular}

Fuente: Elaboración propia.

Los variaciones porcentuales de los ingresos operacionales de las empresas de cada sector; son valores promedio para la serie temporal. Se destacan las empresas de los sectores agropecuario y quími$\mathrm{co}$, por tener las mayores tasas de crecimiento, mostrando esto que pese a que la economía colombiana ha avanzado bastante hacia la terciarización, los sectores primario y secundario aún se mantienen con gran vigencia en el mercado; no obstante que los resultados de entorno macroeconómico no les sean del todo favorables, específicamente en el caso del sector agropecuario, donde se verificó que las empresas responden de manera negativa a las variaciones del PIB y son muy sensibles a la tasa de inflación. 
Variables macroeconómicas e ingresos operacionales de las empresas colombianas... Yánez Contreras, Martha Alicia y Angulo Pico, Grace Margarita

\section{Conclusiones}

El entorno macroeconómico es condicionante del desempeño empresarial de acuerdo al sector y características internas inobservables y/o aleatorias de las firmas.

La evolución de las variables agregadas de la economía colombiana entre 1998-2009, estuvo enmarcada por tres sucesos: en primer lugar, la recesión de finales de los noventa; en segundo, el proceso de recuperación posterior definido por un nuevo entorno político y social; y por último la crisis financiera mundial de finales de 2007. Todos estos acontecimientos impactaron los ingresos operacionales de las empresas colombianas de manera diferente, de acuerdo al sector al que pertenecen. Variables macroeconómicas como tasa de interés y variación del índice de tasa de cambio real, resultaron significativas en los sectores químico y otros servicios; demostrando de esta manera la importancia que tiene el estilo de política monetaria que se geste en el país; porque incrementos de la una u otra variable, afectan negativamente los ingresos de estos sectores, por su gran dependencia a las importaciones y evidentemente por el efecto expulsión que genera sobre la inversión, el aumento del costo del dinero.

La tasa de desempleo resultó significativa en los sectores energía y construcción, debido a la importancia de la demanda interna en los resultados de los mismos, por tanto caídas en los niveles de empleo, significan menos probabilidades de compra de los clientes nacionales. Por su parte, el sector agropecuario mostró gran sensibilidad ante cambios en la tasa de inflación, por la necesidad de mantener bajos precios para competir internacionalmente. Sorpresivamente, los ingresos operativos del sector mostraron una relación inversa con el PIB departamental, debido en gran parte al advenimiento de la terciarización de la economía colombiana, que ha dejado en complejas circunstancias al sector primario del país. No obstante; las mayores variaciones autónomas de los ingresos empresariales, dadas por el estimador de efectos fijos y el componente inobservable de cada firma, estuvieron en las empresas de los sectores agropecuario y químico, demostrando así su vigente importancia, pese al auge del tercer sector. Los ingresos operacionales de las empresas del sector comercio, no resultaron estadísticamente relacionados con las variables macroeconómicas; sin embargo, dados los resultados se infiere que el PIB departamental y la variación del índice de tasa de cambio real influyen en éstos.

Es importante exponer que las variables internas de las firmas fueron significativas en la mayoría de los casos, contribuyendo al ajuste de los modelos. A su vez, el estimador adecuado para los modelos empresariales es el de efectos fijos, porque muestra de manera más precisa la heterogeneidad inobservable de estas unidades transversales, aunque es factible pensar en efectos aleatorios para casos particulares. 


\section{Referencias bibliográficas}

Aguilar, Camila (2003). Impactos de Eventos Exógenos y Sectoriales sobre el Desempeño de las Empresas: Un Análisis para Colombia 1995-2001. Documento CEDE. Universidad de los Andes (31).

Arias Leyva, Andrés F. (2007). El Sector Agropecuario en la Política Macroeconómica del País. Ministerio de Agricultura y Desarrollo Rural, Bogotá D.C.

Audretsch, David B., \& Acs, Zoltan (1994). New Firm Startups, Technology, and Macroeconomic Fluctuations. Small Business Economics, 6 (6), 439-449.

Banco de la República (2006). Impacto del TLC en la Balanza de Pagos de Colombia. Reportes del Emisor. Investigación e Información Económica, Bogotá D.C.

Banco de la República. (2008). Informe sobre Inflación. Banco de la República, Bogotá D.C.

Banco de la República (2009). Informe sobre inflación. Banco de la Repúbluca, Bogotá D.C.

Benavente, José M. \& Külzer, Cintia (2006). Entry and Exit Firms in Chile. Estudios de Economía, 35 (2).

Bonet, Jaime (2006). La Terciarización de las Estructucturas Económicas Regionales en Colombia. Banco de la República. Centro de Estudios Económicos Regionales (CEER). Documentos sobre Economía Regional. (67), 37.

Camacho García, María. O. \& Flamand, Laura (2008). Políticas Gubernamentales para Controlar la Contaminación del Aire en Ciudades Mexicanas. Gestión y Política Pública. Centro de Investigación y Docencia Económica. XVII (2), 261-313.

Cámara Colombiana de la Construcción ( CAMACOL). (2008). Tendencias Macroeconómicas y de la Industria de la Construcción 2008-2009. Informe
Especial del Departamento de Estudios Económicos para la LVI Asamblea Nacional de Afiliados a la Cámara Colombiana de la Construcción (Camacol). Bogotá D.C.

Cámara de Usuarios de Zonas Francas. (2008). Régimen Franco y Sector Químico, Plástico y Petroquímico. Segundo Congreso Nacional de Zonas Francas, Asociación Nacional de Industriales, Bogotá D.C.

Cámaras de Usuarios de Zonas Francas. Asociación Nacional de Industriales (2009). Régimen Franco y Sector Plástico, Químico y Petroquímico. Bogotá D.C.

Colombia. Presidencia de la República. (1991). Constitución Política de Colombia. Bogotá D.C.

Consejo Intergremial de Minería en Colombia (CIMCO); Asociación Colombiana de Petróleo (ACP); Asociación Nacional de Comercio Exterior (ANALDEX); Asociación Nacional de Industriales (ANDI); Cámara Colombiana de Servicios Petroleros (CAMPETROL). (2009). Sector Minero y Petrolero en Colombia. Bogotá D.C.

Corredor Avella, Germán (2008). Política Energética en Colombia. Encuentro Latinoamericano de Economía de la Energía, Observatorio Colombiano de Energía. Universidad Nacional de Colombia, Bogotá D.C.

Fedesarrollo (2008). Tendencia Económica. Informe Mensual, Bogotá D.C.

Fedesarrollo. Centro de Investigación Económica y Social (2012). Impacto socioeconómico de la minería en Colombia. Bogotá D.C.

Huerta Riveros, Patricia, Predaja Rejas, Liliana, Navas López, José E. \& Almódovar M. Paloma (2008). Las Estrategias Corporativas y los Resultados Empresariales: Un Análisis por Tipo de Estrategia Corporativa y Sector industrial en España. Cuadernos de 
Variables macroeconómicas e ingresos operacionales de las empresas colombianas... Yánez Contreras, Martha Alicia y Angulo Pico, Grace Margarita

Administración. Pontificia Universdad Javeriana, 21 (037), 61-71.

Jensen, Michael (1986). Agency Cost or Free Cash Flow, Corporate Finance, and Takeovers. American Economic Review, 76 (2), 323-329.

Langebaek, Andrés. Banco de la República (2008). Determinantes del Crecimiento de las Empresas no Financieras en Colombia: Ley de Gibrat y Otras Teorías. Borradores de Economía. Banco de la República (493).

León León, Josefina (2007). La Estabilidad Macroeconómica y el Papel del Mecanismo de Transmisión de la Política Monetaria. Análisis Económico. Universidad Autónoma Metropolitana, XXII (51), 29-58.

Lizarazu Alanez, Eddy (2006). La Macroeconomía IS-LM. Una Restrospección Teorética Estilizada. Investigación Económica, LXV (256), 103-129.

Long Chen, Hong (2010). Using Financial and Macroeconomic Indicators to Forecast Sales of Large Development and Construction Firms. Journal of Real Estate Finance and Economics, 40 (3).

López Soler, Ramiro (2006). Relaciones entre la Experiencia Exportadora y el Desempeño Productivo de la Industria. Desarrollo y Sociedad. Universidad de los Andes (57), pp. 43-75.

Machin, Stephen, \& Van Reenen, John (1993). Profit Margins and the Business Cycle: Evidence from UK Manufacturing Firms. The Journal of Industrial Economics, 41 (1), pp. 29-50.

Ortiz, Javier (2005). Empresa y Entorno Económico: Una Experiencia del Grupo de Estudios en Economía y Empresa. Revista Universidad EAFIT, 41 (137), pp. 19-35.

Rosas Vega, Gabriel (2007). La Situación de los Sectores Agropecuario y Rural. Cuadernos de Desarrollo Rural. Pontificia Universidad Javeriana (050), 9-17.
Rugeles Chacón, Laura y Francois Jolly Jean (2006). Hacia la Construcción de Modelos Agroempresariales en una Perspectiva Territorial. Cuadernos de Administración. Pontificia Universidad Javeriana, 19 (032), 295-317.

Saldarriaga, Esteban (2006). Determinantes del Sector de la Construcción en Colombia. Ministerio de Ambiente, Vivienda y Desarrollo TerritorialFirst iniciative, Bogotá D.C.

Santoro, Emiliano, \& Gaffeo, Edoardo. (2009). Business Failures, Macroeconomic Risk and the Effect of Recessions on Long-Run Growth: A Panel Cointegration Approach. Journal of Economics and Business (61), pp. 435-452.

Superintendencia de Sociedades de Colombia (2007). Comportamiento del Sector Minero Colombiano. Superintendencia de Sociedades, Grupo de Estadística, Bogotá D.C.

Superintendencia de Sociedades de Colombia (2004). Evolución y Desarrollo del Sector Agroindustrial 2000-2003. Superintendencia de Sociedades de Colombia, Bogotá D.C.

Superintendencia de Sociedades de Colombia (2004). Informe Comportamiento Sectorial 2002-2003. Superintendencia de Sociedades, SIREM, Bogotá D.C.

Wooldridge, Jeffrey (2007). Métodos Avanzados para Datos de Panel. En J. Wooldridge, Introduccion a la Econometria. Un Enfoque Moderno (Segunda ed., pp. 513-540). Madrid: Thomson Editores Spain.

Scitovsky, T. (Abril de 1954). Two Concept of External Economies. The Journal of Political Economy, 62(2), pp. 143-151.

Cardona, M. y Cano, C. (Septiembre de 2005). Análisis Sectorial y Regional del Ciclo de Vida de las Firmas Colombianas en el periodo 1995- 2000: Un modelo de datos de panel. Economía, Sociedad y Territorio, 5(19), pp. 555-593. 